\title{
Time integration error estimation for continuous Galerkin schemes
}

\author{
Stephan Kizio* and Karl Schweizerhof \\ Institut für Mechanik, Universität Karlsruhe
}

\begin{abstract}
The purpose of this contribution is the time integration error estimation for continuous Galerkin schemes applied to the linear semi-discrete equation of motion. A special focus is on the effort for the error estimation for large finite element models. Error estimators for the global time integration error as well as for the local error in the last time interval are presented. The Galerkin formulation in time allows the application of the well-known duality based error estimation techniques for the estimation of the time integration error. The main effort of these error estimators is the computation of the dual solution. In order to diminish the computational effort for solving the dual problem the error estimation is carried out in a reduced modal basis. The relevant modes which have to remain in the basis can be determined via the initial conditions of the dual problem.
\end{abstract}

\section{Local and global error estimation}

For problems in linear elastodynamics usually a semi-discrete approach is chosen which yields a system of coupled ordinary differential equations:

$$
\mathbf{M} \ddot{\mathbf{d}}(t)+\mathbf{C} \dot{\mathbf{d}}(t)+\mathbf{K d}(t)=\mathbf{F}(t)
$$

The continuous Galerkin method with quadratic polynomials is then well suited for the solution of this semi-discrete system. In order to choose appropriate time step sizes error estimators are needed. In [6] an adaptive time stepping scheme is presented which is based on the estimation of the local and the global time integration error. Therefore local and global time integration error estimators based on the adjoint or dual problem can be used [1], [2],[3], [5],[6]. The dual problem is a backward problem in time with initial conditions at the current time $t_{m}$ and can be derived from the primal problem via integration by parts. For the error estimation the residual $\mathbf{R}$ of the primal problem is weighted with the solution of the homogeneous dual problem:

$$
\mathbf{M} \ddot{\mathbf{z}}(t)-\mathbf{C} \dot{\mathbf{z}}(t)+\mathbf{K z}(t)=\mathbf{0} \text { with } \mathbf{z}\left(t_{m}\right)=\mathbf{z}_{m} \text { and } \dot{\mathbf{z}}\left(t_{m}\right)=\dot{\mathbf{z}}_{m}
$$

Then the computed error quantity only depends on the initial conditions of the dual problem [1],[3],[6]. Here we restrict ourselves on the estimation of the error at a particular degree of freedom $i$. For the arbitrary choice of $\mathbf{z}_{m}=\mathbf{0}$ the corresponding initial velocities for the dual problem are:

$$
\dot{\mathbf{z}}\left(t_{m}\right)=-\mathbf{M}^{-\mathbf{1}} \mathbf{1} \text { with } \mathbf{1}(i)=1 \text { for } i=d \text { and } \mathbf{1}(i)=0 \text { for } i \neq d .
$$

Introducing these initial conditions into the weighted residual form of the error equation yields the following error representations of the local error $e_{l, i}\left(t_{m}\right)$ of the last time step and the accumulated global error $e_{g, i}\left(t_{m}\right)$ of all time steps [6]

$$
e_{l, i}\left(t_{m}\right)=\int_{t_{m-1}}^{t_{m}} \mathbf{z} \cdot \mathbf{R} d t \text { and } e_{g, i}\left(t_{m}\right)=\int_{0}^{t_{m}} \mathbf{z} \cdot \mathbf{R} d t
$$

The error estimators are now based on the direct computation of equation (4), i.e. the numerical solution of the dual problem has to be determined. With the transformation $t^{*}=t_{m}-t$ the dual problem can be formulated like the homogeneous primal problem. Thus usually the dual problem is solved using the same time integration scheme as for the primal problem. Since for the estimation of the local error the weighted residual only has to be computed in the corresponding time interval, the numerical effort for the local error estimation is fairly small. On the other hand for global error estimation at each time at which the error shall be estimated a complete backward integration of the dual problem has to be carried out. Thus the computational effort for the dual solution is much higher than for the primal solution. This is a considerable drawback for the practical application of the global error estimator especially for large finite element models. In addition for global error estimation the whole primal solution - displacements and velocities - has to be stored. Another problem is that due to the phase error of the used time integration scheme the time step size of the primal problem may not be suitable for the computation of the dual problem since for the error estimation the dual solution should be determined as accurately as possible. As a result the efficiency of the global error estimation strongly depends on the appropriate time step size.

\footnotetext{
* Corresponding author: e-mail: kizio@ifm.uni-karlsruhe.de, Phone: +49 721 608 3252, Fax: +49721 6087990
} 


\section{Global error estimation in a reduced modal basis}

In order to reduce the computational effort of the global error estimation an alternative error estimator based on the modal decomposition of the error equation is proposed. The solution of the dual problem can be computed exactly in the complete modal basis, i.e. no time integration error is introduced into the dual problem. It should be noted that no complete modal decomposition of the problem shall be carried out since then no time integration of the semi-discrete equation would be necessary. For the error estimation only a strongly reduced modal basis is needed which can be determined from the intial conditions of the dual problem.

The modal decomposition of the primal and the dual problem yields an error representation in modal coordinates where the modal residual of the primal problem is tested with the modal solution $\mathbf{q}_{d}$ of the dual problem

$$
e_{g, i}\left(t_{m}\right)=\int_{0}^{t_{m}} \mathbf{q}_{d} \cdot\left(\boldsymbol{\Phi}^{T} \mathbf{F}-\boldsymbol{\Phi}^{T} \mathbf{M} \boldsymbol{\Phi} \ddot{\mathbf{q}}_{p}-\boldsymbol{\Phi}^{T} \mathbf{C} \boldsymbol{\Phi} \dot{\mathbf{q}}_{p}-\boldsymbol{\Phi}^{T} \mathbf{K} \boldsymbol{\Phi} \mathbf{q}_{p}\right) d t=\sum_{j=1}^{n_{\text {mod }}}\left(\int_{0}^{t_{m}} q_{d, j} \cdot R_{j} d t\right) .
$$

In this representation all equations are decoupled and so the error can be split into the sum of the errors of the $n_{\bmod }$ modes. If all modes are scaled such that all modal masses are 1 the initial conditions of the dual problem read

$$
\dot{\mathbf{q}}_{d}\left(t_{m}\right)=\boldsymbol{\Phi}^{T}(-\mathbf{1})=-\left[\begin{array}{c}
X_{1, i} \\
\vdots \\
X_{n, i}
\end{array}\right]
$$

i.e. no inversion of the massmatrix as in equation (3) is needed.

The first step of constructing the reduced modal basis is based on to the fact that for practical problems the solution is dominated by the lower natural frequencies. Therefore only the first $n_{l o w}<n_{d f}$ natural frequencies need to be determined.

The dual problem provides further information on the relevant modes which have to be considered for the error estimation. According to equation (5) the amplitudes of the dual solution $q_{\max , j}$ of each mode can be taken as an indicator of the possible influence of the corresponding mode $j$ on the time integration error at the particular node $i$. These amplitudes can be computed directly from the initial velocities according to equation (6):

$$
q_{\max , j}=\left|\frac{\dot{q}_{d, j}\left(t_{m}\right)}{\omega_{j} \sqrt{1-\xi_{j}^{2}}}\right| \text { with } \xi_{j}=\frac{c_{j}}{2 \omega_{j} m_{j}}
$$

Now the largest amplitude is defined as the reference value $q_{r e f}=\max \left(q_{d, \max }\right)$ and only modes for which holds

$$
q_{d, \max , j} \geq c q_{\text {ref }} \text { with } 0<c<1
$$

remain in the modal basis for the error estimation. Since the time integration error also depends on the modal residual of the primal problem the factor $c$ in equation (8) should not be chosen too small. Numerical examples show that good results are obtained with $0.1<c<0.5$.

\section{Conclusions}

We presented a global time integration error estimator for continuous Galerkin schemes in a reduced modal basis. The main effort of the proposed procedure is the construction of this reduced modal basis respectively the initial conditions of the dual problem. A similar approach was chosen in [4] for the model reduction in structural dynamics. In the linear case the reduced modal basis can be determined directly from the initial conditions of the dual problem in modal coordinates. For the evaluation of the reduced error equation no time integration of the dual problem is needed which is the main effort of the classical time integration error estimation described in section 1. Another advantage of the proposed global error estimator is that in contrast to the classical approach the primal problem has only to be stored in the reduced modal basis.

\section{References}

[1] W. Bangert. Adaptive Finite-Elemente-Methoden zur Lösung der Wellengleichung mit Anwendung in der Physik der Sonne. Diplomarbeit, Ruprecht-Karls-Universität Heidelberg, 1998.

[2] D. Estep. A posteriori error bounds and global error control for approximations of ordinary differential equations. SIAM Journal of Numer. Analysis, 32(1):1-48, 1995.

[3] A. Maute. Fehlerkontrolle bei Finite-Elemente-Methoden in der linearen Strukturdynamik. Dissertation, Universität Stuttgart, 2001.

[4] M. Meyer. Reduktionsmethoden zur Simulation des aeroelastischen Verhaltens von Windkraftanlagen. Dissertation, Universität Braunschweig, 2002.

[5] J. Neumann. Anwendung von adaptiven Finite Element Algorithmen auf Probleme der Strukturdynamik. Dissertation, Universität Karlsruhe, 2004.

[6] K. Schweizerhof, J. Neumann, and S. Kizio. On time integration error estimation and adaptive time stepping in structural dynamics. Proc. Appl. Math. Mech., 4:35-38, 2004. 